\title{
Early activation of quorum sensing in Pseudomonas aeruginosa reveals the architecture of a complex regulon Martin Schuster ${ }^{1,2}$ and E Peter Greenberg*1
} Address: ${ }^{1}$ Department of Microbiology, University of Washington, Box 357242, 1959 NE Pacific St., Seattle, WA 98195, USA and ${ }^{2}$ Current address:
Department of Microbiology, Oregon State University, 220 Nash Hall, Corvallis, OR 97331, USA

Email: Martin Schuster - martin.schuster@oregonstate.edu; E Peter Greenberg* - epgreen@u.washington.edu

* Corresponding author

Published: 22 August 2007

BMC Genomics 2007, 8:287 doi:10.1 |86/|47|-2164-8-287
Received: 22 March 2007

Accepted: 22 August 2007

This article is available from: http://www.biomedcentral.com//47/-2/64/8/287

(c) 2007 Schuster and Greenberg; licensee BioMed Central Ltd.

This is an Open Access article distributed under the terms of the Creative Commons Attribution License (http://creativecommons.org/licenses/by/2.0), which permits unrestricted use, distribution, and reproduction in any medium, provided the original work is properly cited.

\begin{abstract}
Background: Quorum-sensing regulation of gene expression in Pseudomonas aeruginosa is complex. Two interconnected acyl-homoserine lactone (acyl-HSL) signal-receptor pairs, 3-oxododecanoyl-HSL-LasR and butanoyl-HSL-RhIR, regulate more than 300 genes. The induction of most of the genes is delayed during growth of $P$. aeruginosa in complex medium, cannot be advanced by addition of exogenous signal, and requires additional regulatory components. Many of these late genes can be induced by addition of signals early by using specific media conditions. While several factors super-regulate the quorum receptors, others may co-regulate target promoters or may affect expression posttranscriptionally.

Results: To better understand the contributions of super-regulation and co-regulation to quorumsensing gene expression, and to better understand the general structure of the quorum sensing network, we ectopically expressed the two receptors (in the presence of their cognate signals) and another component that affects quorum sensing, the stationary phase sigma factor RpoS, early in growth. We determined the effect on target gene expression by microarray and real-time PCR analysis. Our results show that many target genes (e.g. las $B$ and $h c n A B C$ ) are directly responsive to receptor protein levels. Most genes (e.g. lasA, lecA, and phnAB), however, are not significantly affected, although at least some of these genes are directly regulated by quorum sensing. The majority of promoters advanced by RhIR appeared to be regulated directly, which allowed us to build a RhIR consensus sequence.

Conclusion: The direct responsiveness of many quorum sensing target genes to receptor protein levels early in growth confirms the role of super-regulation in quorum sensing gene expression. The observation that the induction of most target genes is not affected by signal or receptor protein levels indicates that either target promoters are co-regulated by other transcription factors, or that expression is controlled posttranscriptionally. This architecture permits the integration of multiple signaling pathways resulting in quorum responses that require a "quorum" but are otherwise highly adaptable and receptive to environmental conditions.
\end{abstract}




\section{Background}

In Pseudomonas aeruginosa, two acyl-homoserine lactone quorum sensing (acyl-HSL QS) systems, LasR-LasI and RhlR-RhlI, control the expression of partially overlapping sets of genes. Many of the regulated genes are implicated in virulence and biofilm formation of this opportunistic pathogen. LasI and RhlI are enzymes that synthesize the acyl-HSL signals 3-oxo-dodecanoyl (3OC12)-HSL and butanoyl (C4)-HSL, respectively. LasR and RhlR are receptors that specifically bind the signal generated by their cognate synthases to regulate transcription of target genes [1-5]. Three independent transcriptome analyses identified several hundred such genes [6-8]. The numbers of identified genes and their expression patterns varied among studies depending on experimental conditions and statistical criteria used. In our study [8] we noted that the expression of most quorum-controlled genes increased in the stationary phase of growth and could not be advanced to logarithmic phase by the addition of exogenous signal, confirming earlier observations with individual quorum-controlled genes [9-11]. This suggested that the activation of most quorum-controlled genes is not triggered by the accumulation of signal, and seems to require additional factors.

In accordance with this hypothesis, several other regulatory systems such as the GacAS-RsmAZ two-component signal transduction pathway [12-14], the stationary phase sigma factor RpoS $[9,15]$, and MvfR (PqsR)-quinolone signaling $[16,17]$, have been shown to influence quorumcontrolled gene expression in $P$. aeruginosa. They are thought to affect las or $r h l$ mediated QS at two distinct levels of signal integration, either at the level of the regulators LasR and RhlR themselves, or at the level of complex QS target promoters [18] (Fig. 1). The stationary phase sigma factor RpoS, for example, appears to affect QS gene expression at both levels [15]. Furthermore, a recent study showed that there is an inhibitory substance (or substances) in complex medium which delays the expression of many quorum-controlled genes [19], but it is not clear at which level this occurs. Because transcripts of las $R, r h l R$, and $r p o S$ increase at the onset of stationary phase in concert with activation of most quorum-controlled genes $[8,15]$, it is possible that the abundance of the encoded proteins is critical for target gene induction. On the other hand, we predict that those late genes for which these regulators are not limiting early in growth are co-regulated by other factors at the target promoter level. To better understand the network structure of the QS regulon, we expressed LasR, RhlR, and RpoS from a heterologous promoter in logarithmic phase and compared quorum-controlled gene expression in the corresponding strains to that of the wildtype by using DNA microarrays.

\section{Results and discussion \\ LasR, RhIR and RpoS levels increase in the stationary phase of growth}

Our previous transcriptome analyses $[8,15]$, in addition to other studies with reporter fusions [20-22], showed that the transcript levels of lasR, rhlR, and $r p o S$ increase at the transition from logarithmic to stationary phase during batch culture growth of $P$. aeruginosa. Immunoblotting with LasR, RhlR, and RpoS-specific antisera showed that this expression pattern correlates well with the respective protein levels (Fig. 2).

\section{Expression strategy}

If LasR, RhlR or RpoS levels were limiting the expression of QS-controlled genes during logarithmic growth of wildtype $P$. aeruginosa, early expression of these factors should advance quorum-controlled gene expression from stationary to logarithmic phase. To test this hypothesis, we constructed strains for regulatable expression of LasR, RhlR, and RpoS. Each allele, under the control of an arabinose-inducible araBAD promoter, was inserted in single copy into a neutral site of the chromosome of a lasR, rhlR mutant or an rpoS mutant, respectively. This arrangement allowed the careful titration of expression levels in logarithmic phase such that they matched those of the wildtype in stationary phase. For LasR and RhlR, it also allowed us to assess the impact of each regulator on gene expression independently and uncoupled from the QS hierarchy, where LasR-LasI is required for RhlR-RhlI expression $[20,23,24]$. We have thoroughly validated this approach by monitoring target gene expression and regulator protein levels in the engineered strains and in the parent strain throughout growth (see below).

\section{Many QS genes can be advanced by early expression of LasR, RhIR, or RpoS, but not by signal addition}

Early expression of LasR and RhlR each resulted in the premature induction of 125 and 127 genes, respectively, during growth of $P$. aeruginosa in culture $(\mathrm{p}<0.001$ using Cyber-T analysis). Sixty-seven of the genes induced by LasR and 55 of the genes induced by RhlR are among the set of 315 quorum-induced genes we identified in a previous study [8] (Fig. 3A and Additional file 1). Early expression of RpoS resulted in the premature induction of 124 genes. Sixty-one of these genes were identified as quorumcontrolled, and 36 are among the genes previously identified as RpoS-induced [15]. Thus, early expression of any of the three proteins revealed genes that are not among the previously characterized QS and RpoS regulons. Although this finding warrants further investigation, for the purpose of this study we focused on the set of 315 previously identified and well characterized quorum-regulated genes [8].

A heat map shows that the early expression of LasR, RhlR, and Rpos induced many genes in logarithmic phase to 


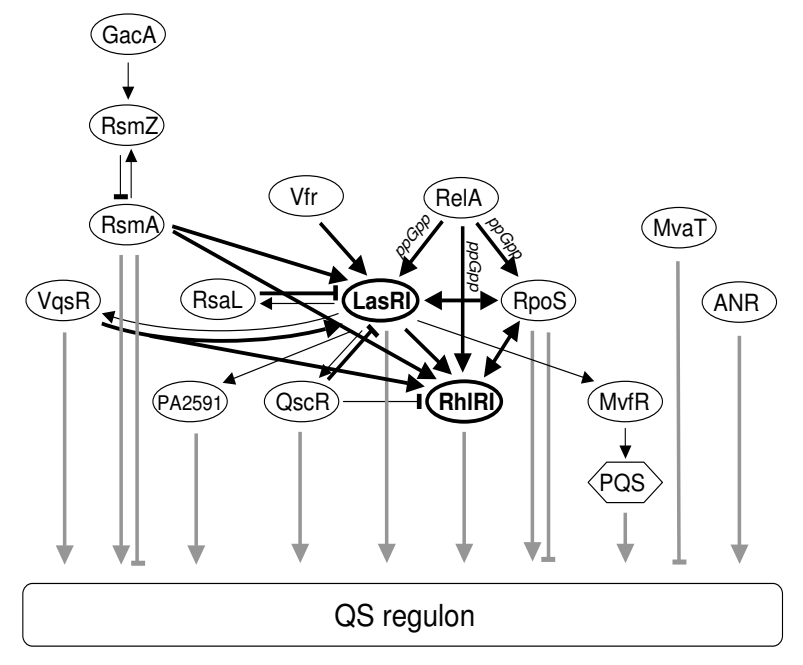

Figure I

The relationship between QS and other regulatory pathways. The QS regulon is defined as the collection of genes activated by $30 \mathrm{CI} 2-\mathrm{HSL}-$ LasR or C4-HSL-RhIR, directly or indirectly. The different regulatory pathways shown affect the expression of overlapping subsets within the QS regulon. Effects on signal synthase and receptor expression have not been detailed separately. The bold black lines indicate superregulation of LasR-Lasl and RhIR-Rhll. The bold grey lines indicate co-regulation of target genes. PA259। is one of several predicted transcriptional regulators activated by las QS. This figure has been modified from [18].

levels close to those of the wildtype in stationary phase (Fig. 3B). Nineteen genes were also activated by the addition of 3OC12-HSL and C4-HSL signals alone, but their induction levels were comparatively low and all of them could be induced further by early expression of RhlR (Fig. 3). Early expression of RpoS often only partially activated those genes that were more highly activated by LasR or RhlR. Because RpoS also has a small effect on LasR and RhlR expression [15], it appears that RpoS is capable of advancing many genes only indirectly through LasR and RhlR. Overall, there was a good correlation between the genes induced by early expression of LasR or RhlR, and their signal specificity determined by addition of 3OC12HSL or both 3OC12-HSL and C4-HSL to a signal generation mutant [8]. For example, a cluster of $r h l$-specific genes was advanced by C4-HSL-RhlR but not by 3OC12-HSLLasR (Fig. 3C), whereas several las-specific genes were advanced by 3OC12-HSL-LasR but not C4-HSL-RhlR (Fig. 3D). However, there were exceptions. PA5481 and PA5482, for example, can be advanced by 3OC12-HSLLasR but not C4-HSL-RhlR (Fig. 3D) although in a signal synthesis mutant they responded better to both signals

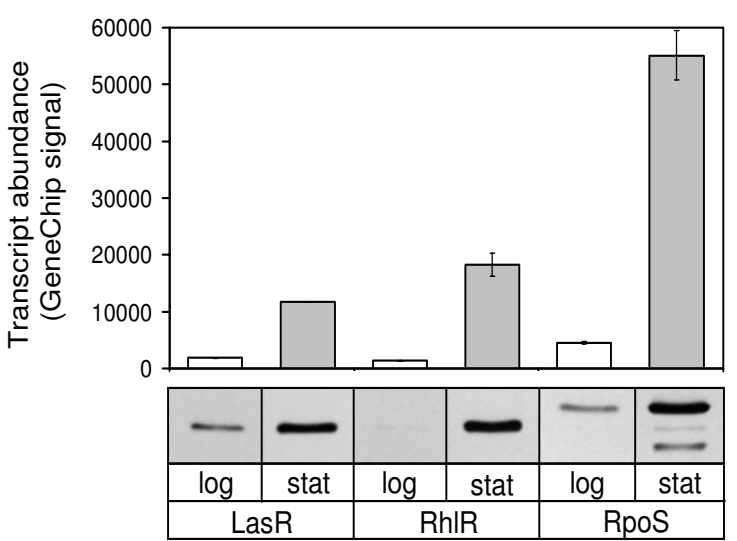

\section{Figure 2}

Transcript and protein levels of LasR, RhIR, and RpoS during growth of $\boldsymbol{P}$. aeruginosa. Top panel, Pseudomonas GeneChip signal levels; bottom panel, immunoblots of clarified lysates with LasR, RhIR, and RpoS specific antibodies.

The two low molecular weight bands detected by RpoS antibody in stationary phase lysates represent partially degraded RpoS.

than to 3OC12-HSL alone [8]. Such genes may require the binding of both regulators to their promoters for activation, or they may require additional factors that are themselves under the control of 3OC12-HSL-LasR.

Among the genes significantly induced by early expression of LasR, RhlR, or RpoS were $47 \%$ of all genes activated early by addition of signals to cells in conditioned medium [19]. The large majority of this subset was induced by early expression of RhlR. RhlR was one of the genes triggered by media conditioning [19] and it also induced in minimal medium [24]. Thus, the advancement of QS in conditioned medium appears to be partially mediated through super-regulation of RhlR.

\section{Many other QS genes cannot be advanced significantly by early expression of LasR or RhIR}

Most quorum-controlled genes showed little to no activation by LasR, RhlR or RpoS expression in logarithmic phase. One-hundred and twenty of these genes are late genes. Their quorum-dependent (and in most cases RpoSindependent) induction in the wildtype strain is delayed until stationary phase (Additional file 2). These late genes could not be advanced although several are predicted to be directly activated by LasR or RhlR as they possess conserved sequence elements, so-called las-rhl boxes, in their promoter regions $[7,8,11]$. Thus it appears that 3 OC12- 
A

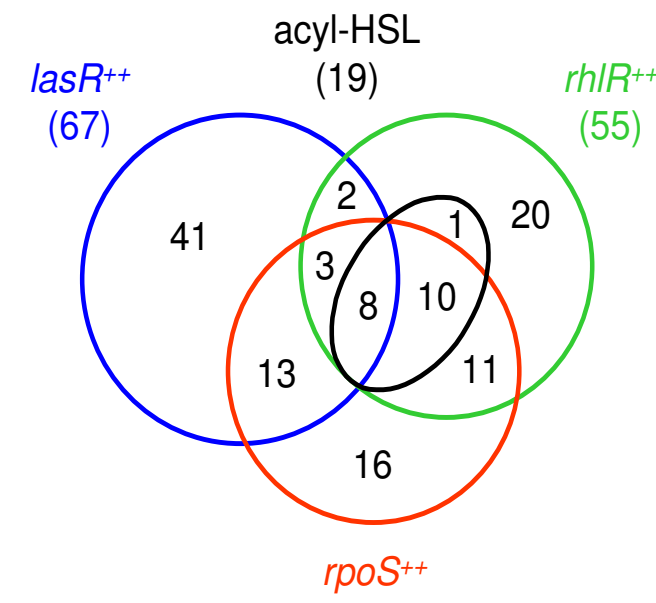

$(61)$
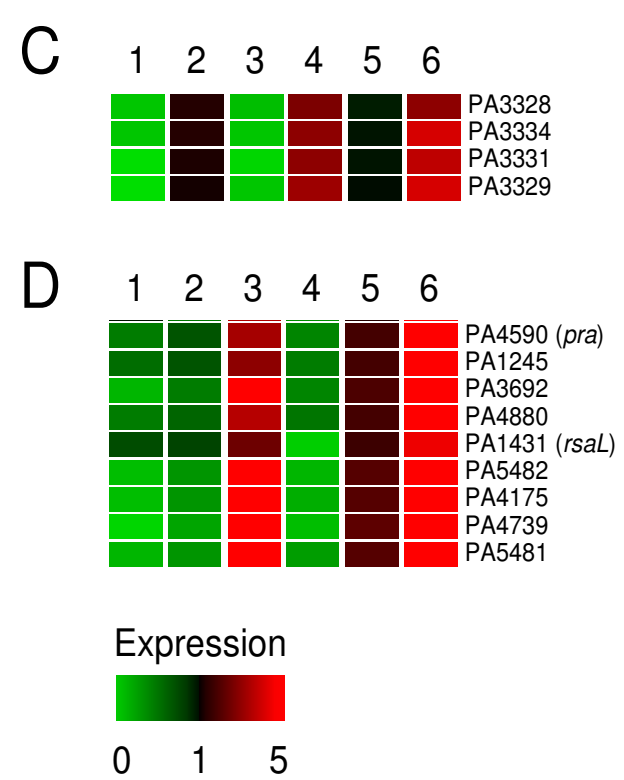

B

(C)
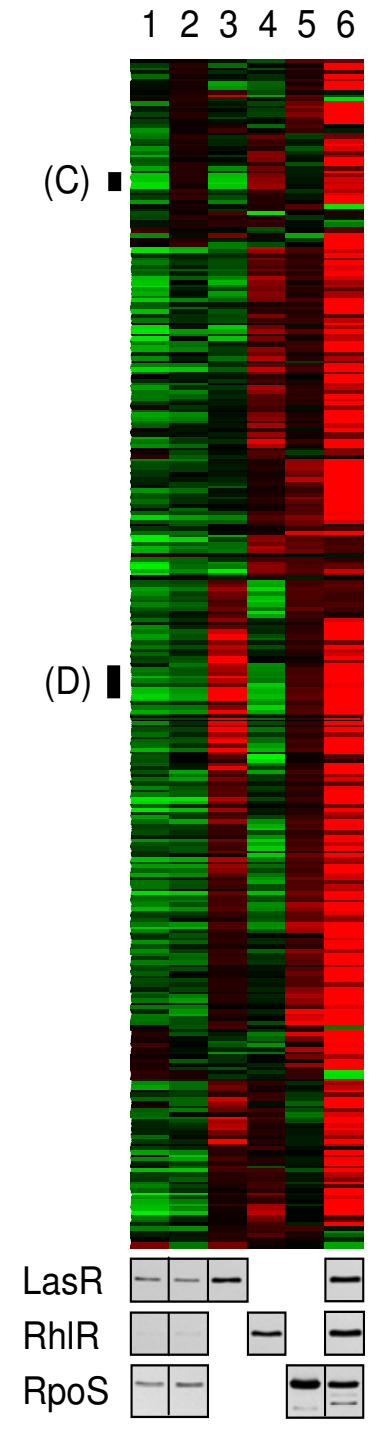

\section{Figure 3}

Advancement of quorum-controlled gene expression. A. Venn diagram showing the numbers of genes significantly induced by acyl-HSL addition or by early expression of LasR, RhIR, or RpoS. Comparison was relative to the PAOI parent strain without exogenous signal in logarithmic phase. B. Top panel, $P$. aeruginosa transcript profiles (absolute GeneChip signal levels) of all previously identified 315 quorum-activated genes [8] under various growth and expression conditions. Strains were incubated in the presence of arabinose with or without $2 \mu \mathrm{M} 30 \mathrm{CI}$-HSL and $10 \mu \mathrm{M}$ C4-HSL. Lane I, PAOI parent without signals in logarithmic phase (50 mM arabinose); lane 2, PAOI parent with signals in logarithmic phase (50 mM arabinose); lane 3, lasR, rhIR mutant expressing las $R$ with signals in logarithmic phase (7.5 mM arabinose); lane 4, las $R$, rhlR mutant expressing $r / R$ with signals in logarithmic phase ( $50 \mathrm{mM}$ arabinose); lane $5, r p o S$ mutant expressing rpoS with signals in logarithmic phase ( $25 \mathrm{mM}$ arabinose); lane 6, wildtype without signals in stationary phase (I mM arabinose). Bottom panel, immunoblots showing the corresponding protein levels of LasR, RhIR and RpoS under the conditions described above. C and D, Transcript profiles of individual genes enlarged from B. 
HSL-LasR, C4-HSL-RhlR, and RpoS levels are not limiting for the activation of many quorum-controlled genes.

To demonstrate that late quorum-controlled genes can still be activated in stationary phase using our engineered expression strains, and to confirm our microarray results, we measured the logarithmic and stationary phase transcript profiles of several quorum-controlled genes by realtime PCR. The genes lasA, PA0179, PA2939, and rhlA, which showed little to no quorum activation in logarithmic phase, could be activated in stationary phase cultures of strains expressing las $R$ or $r h l R$ from the pBAD promoter (Fig. 4). Both lasA and rhlA possess a las-rhl box $[7,8,25]$. PA3905 is an example of one of the few early genes that are induced fully in the wildtype strain in logarithmic phase. Ectopic expression of lasR resulted in a similar level of induction in stationary phase.

As indicated above, there was generally a good correlation between regulator specificity and acyl-HSL signal specificity (as determined by early expression of LasR or RhlR in a signal receptor mutant and by addition of 3OC12-HSL or both signals to a signal synthesis mutant, respectively). An exception is PA0179, which was 3OC12-HSL specific [8] but responded to both LasR and RhlR expression in logarithmic phase (Fig. 4). This suggests that RhlR-C4HSL is capable of activating PA0179, but this effect is masked in the signal generation mutant because 3OC12HSL-LasR is required for RhlR expression and 3OC12HSL-LasR already saturates the PA0179 promoter. Hence, our expression strategy provides a different assessment of QS promoter specificity because it functions independently of the QS cascade.

\section{Direct versus indirect regulation}

We hypothesized that most genes advanced by QS are directly activated by QS. If this hypothesis is true, then we should be able to identify conserved sequence elements, so-called las-rhl boxes, upstream of these genes. In our previous study, we identified las-rhl box like sequences in 40 out of the 168 predicted quorum-controlled promoters [8]. Many of these promoters are associated with QS advanced genes. Of the 53 predicted promoters advanced by LasR, 16 contain a las-rhl box-like sequence, and of the 26 predicted promoters advanced by RhlR, 17 contain a las-rhl box-like sequence. Thus, it appears that genes advanced by RhIR are for the most part also directly regulated by this transcription factor, whereas genes advanced by LasR are mostly indirectly regulated (i.e. genes directly regulated by LasR are more evenly distributed among the genes that can and cannot be advanced). However, our data do not provide evidence for indirect regulation by Las $R$ via a regulatory cascade. Candidate regulatory genes $m v f R$, PA2591, and $v q s R$, which are under direct transcrip- tional control by LasR $[7,17,26,27]$, are not among the genes activated early (Additional file 1).

Our previous search of las-rhl box like sequences did not take into account differences between las-specific and rhlspecific binding sites. It is therefore possible that we missed several actual LasR or RhlR binding sites. Our identification of a subset of genes advanced by either LasR or RhlR in this study afforded the opportunity for a $d e$ novo, unbiased search for separate LasR and RhlR consensus sequences. We selected those genes most highly induced by LasR alone or by RhlR alone and subjected the presumed upstream regulatory sequences to the pattern discovery algorithm CONSENSUS [28]. A sequence pattern that resembles known binding sites was identified for RhlR-advanced but not for LasR-advanced promoters (Fig. 5). This result confirms the notion that many genes advanced by RhlR are activated directly. Most genes advanced by LasR may be activated indirectly as mentioned above, or LasR binding sites may be more complex. Our recent binding studies with purified LasR support the latter. We noticed a general lack of sequence conservation and identified two subpopulations of las-specific binding sequences based on their distinct interactions with LasR [29]. Such heterogeneity undoubtedly complicates the identification of a single consensus sequence. Further biochemical characterization of a larger number of binding sites will be required for a more elaborate sequence alignment. Our alignment of RhlR sequences shows the conserved $\mathrm{CT}\left(\mathrm{N}_{12}\right)$ AG motif recognized previously $[7,8,11,30]$, and implies several other conserved nucleotides (Fig. 5). Similarity to the original las-rhl box sequence alignment is high, reflecting a bias towards rhlresponsive genes in the original alignment [8].

In a second step, the matrix generated with CONSENSUS was used to search the upstream regions of all previously identified quorum-controlled genes. Fourty-two predicted QS promoters showed a significant match. Not surprisingly, most sequences had also been identified in our previous study [8]. In addition, we identified sequences with similarity to the RhlR consensus upstream of the following genes: PA2081, PA2146, PA3361, PA4141, PA4217 (qsc132), and PA5356 ( $g l c C)$. Of these, the latter three were found to be advanced by RhlR.

\section{Conclusion}

When $P$. aeruginosa is grown in complex medium, LasR, RhlR and RpoS protein levels increase in stationary phase (Fig. 2), correlating with the activation of most quorumcontrolled genes. Our approach to investigating the advancement of quorum sensing gene expression was to adjust the expression levels of regulators in log-phase cells to match the levels in stationary phase. This is an overexpression strategy in which proteins are maintained within 


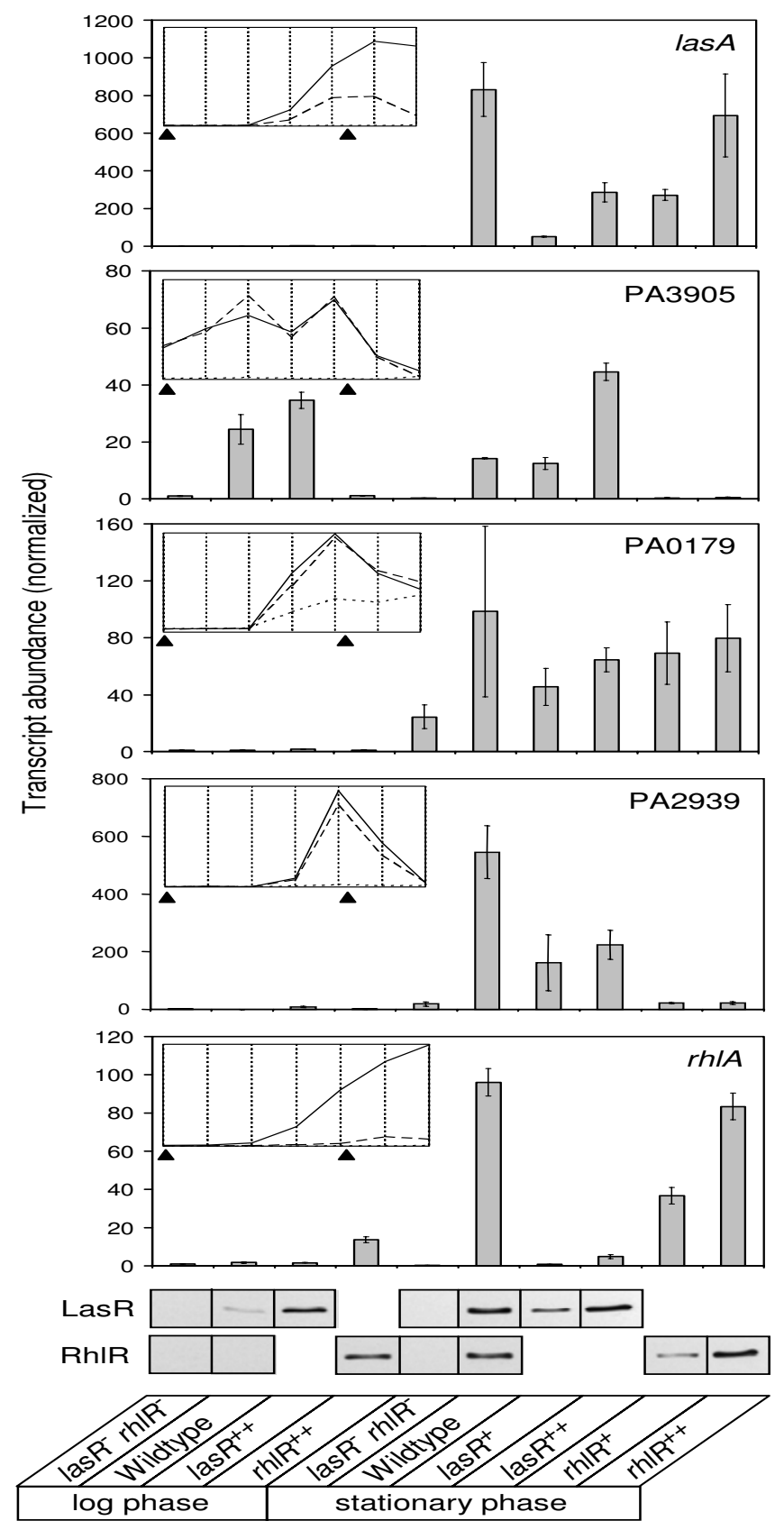

Figure 4

Effect of acyl-HSL addition or early expression of LasR or RhIR on the transcription of selected $P$. aeruginosa quorum-controlled genes in logarithmic and stationary phase. Top panels, transcript abundances as determined by real-time PCR normalized to the transcript levels of the las $R$, rhIR mutant in logarithmic phase. Error bars indicate standard errors of the mean. Concentrations of arabinose added to the cultures were as follows (from left to right, in mM): $50,50,7.5$, 50 , I.0, I.0, 0.I , 0.4, 0.4, I.6. For comparison, the insets in each panel show signal specificities previously determined in a lasl, rhll signal generation mutant without exogenous signal (dotted line), with $2 \mu \mathrm{M} 3 \mathrm{OCI} 2-\mathrm{HSL}$ (dashed line), or with both $2 \mu \mathrm{M}$ 3OCI2-HSL and $10 \mu \mathrm{M}$ C4-HSL (solid line) [8]. The black arrows indicate positions on the growth curve at which the corresponding cultures for real-time PCR analysis were harvested (left arrow, $O D_{600}=0.2$; right arrow, $\mathrm{OD}_{600}=2$ ). Bottom panel, immunoblots showing the respective protein levels of Las $R$ and RhIR. 
A

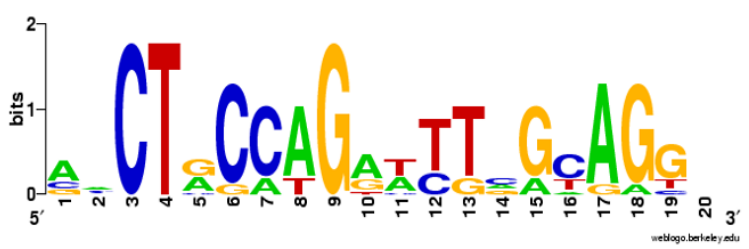

B

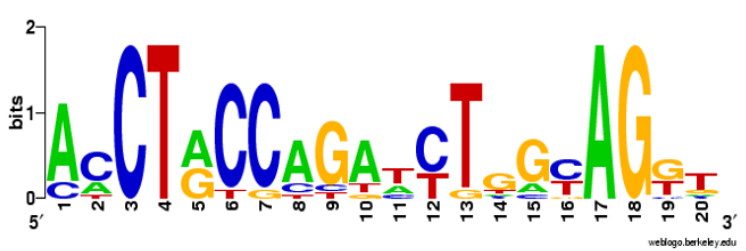

Figure 5

Graphical representation of sequence alignments. (A)

RhIR consensus based on the weight matrix of sequences identified upstream of promoters most highly induced by RhIR early in growth. (B) las-rhl box consensus from our previous study [8]. The las-rhl box alignment is based on II previously identified sequences presumed to bind either LasR, RhIR or both. The overall height of the stack indicates sequence conservation, while the height of symbols within the stack indicates the relative frequency of each nucleic acid.

physiological levels known to exist in P. aeruginosa. Many QS genes could be activated in logarithmic phase by the early expression of LasR, RhlR, and RpoS, but not by signal addition. Thus, the levels of these three proteins can be critical in modulating the quorum-response in $P$. aeruginosa. Several of the genes were induced by more than one regulator, confirming overlapping specificities and co-regulation. Genes that directly and exclusively respond to QS are likely among the subset of genes whose maximal expression can be advanced by increased expression of LasR or RhlR during logarithmic phase. In fact, our results suggested that most genes advanced by RhlR are directly regulated by this transcription factor, allowing us to build a RhlR consensus sequence.

Many other QS genes fail to be advanced by 3OC12-HSLLasR and C4-HSL-RhlR, although it has been shown that some of these genes are directly regulated by these signal and response systems. This confirms and extends a recent observation for an individual gene, rhlA, which is not significantly activated in logarithmic phase even when C4HSL-RhlR is present [31]. These results suggest that the corresponding promoters are co-regulated by other tran- scription factors, likely constituting a network motif known as a multi-input dense overlapping regulon [32]. Some of the regulatory inputs may affect translation rather than transcription, as is the case for small regulatory RNAs that modulate quorum sensing gene expression $[33,34]$. The overall topology allows for specific responses to a multitude of signals, and may provide the basis for the exceptional environmental adaptability of $P$. aeruginosa. It also provides a simple explanation for the seemingly discordant sets of quorum-controlled genes identified by two separate groups under different culture conditions $[7,8,35]$.

Taken together, our results indicate that co-regulation of target genes is an important, and perhaps predominant, feature of the $P$. aeruginosa QS network in addition to the well established super-regulation of the central components, LasR-LasI and RhlR-RhlI. Thus, a thorough understanding of the QS network will necessitate a comprehensive analysis of target promoter architecture, which should include the global identification of transcription factor binding sites. Technologies such as ChIPchip, chromatin immunoprecipitation and microarray analysis [36], make this approach feasible.

\section{Methods \\ Bacterial strains, plasmids, and culture conditions}

Bacterial strains and plasmids are shown in Table 1. For plasmid and strain constructions, bacteria were grown in Luria-Bertani broth (LB). Where appropriate, antibiotics were added to maintain plasmids and to select for recombination or integration events. For transcript profiling experiments, $P$. aeruginosa strains were grown in $250 \mathrm{ml}$ flasks containing $50 \mathrm{ml}$ LB buffered with $50 \mathrm{mM} 3-(\mathrm{N}-$ morpholino) propanesulphonic acid, $\mathrm{pH} 7.0$, at $37^{\circ} \mathrm{C}$ with vigorous shaking. Inocula were from mid-logarithmic phase cultures. The initial optical densities $\left(\mathrm{OD}_{600}\right)$ were 0.02. Synthetic 3OC12-HSL and C4-HSL (2 and 10 $\mu \mathrm{M}$ final concentrations, respectively) and L-arabinose ( 0.4 to $50 \mathrm{mM}$ final concentration) were added to cultures at the time of inoculation as indicated. Different concentrations of arabinose were necessary to induce each individual promoter to the appropriate level.

Strains for regulatable expression of LasR, RhlR, and RpoS were constructed as follows: Alleles of lasR, $r h l R$, and $r p o S$ were placed under control of an arabinose-inducible promoter and inserted in single copy into the chromosome of $P$. aeruginosa using a specialized integration-proficient plasmid system $[37,38]$. The strains contained mutations in the respective chromosomal loci; i.e. las $R$ or $r h l R$ were expressed in PAO lasR $r h l R$, and $r p o S$ was expressed in PAO rpos. Because of constraints with antibiotic resistance markers, las $R$ and $r h l R$ expression constructs were first introduced into an isogenic PAO rhlR single mutant. To 
Table I: Bacterial strains and plasmids

\begin{tabular}{|c|c|c|}
\hline Strain or plasmid & Relevant property & Reference or origin \\
\hline \multicolumn{3}{|l|}{ Escherichia coli } \\
\hline \multirow[t]{3}{*}{$\mathrm{DH} 5 \alpha$} & F-, $\phi 80 \mathrm{dlacZ} \Delta \mathrm{MI} 5 \Delta($ lacZYA-argF)UI69 & Invitrogen \\
\hline & deoR recAl endAI hsdRI7( $\left.\mathrm{rk}^{-}, \mathrm{mk}^{+}\right)$ & \\
\hline & phoA supE44 $\lambda$ - thi-I gyrA96 relA I & \\
\hline SI7-I & recA pro hsdR RP4-2-Tc::Mu-Km::Tn7 & {$[45]$} \\
\hline \multicolumn{3}{|c|}{ Pseudomonas aeruginosa } \\
\hline PAOI & Wild type & {$[46]$} \\
\hline PAOI $\Delta$ lasR & PAOI derivative; $\Delta l a s R:: T c^{R}$ & [47] \\
\hline $\mathrm{PAOI} \Delta r h I R$ & PAOI derivative; $\Delta r h I R:: G m^{R}$ & [47] \\
\hline \multirow[t]{3}{*}{ PAOI $\Delta$ rhIR $\triangle$ lasR } & & PAOI derivative; \\
\hline & & $\Delta r h / R:: \mathrm{Gm}^{\mathrm{R}}$ \\
\hline & & $\Delta$ lasR::TcR \\
\hline \multicolumn{3}{|l|}{ [47] } \\
\hline PAO-rpoS & PAOI derivative; $r p o S:: \mathrm{Gm}^{R}$ & {$[15]$} \\
\hline MSCIOO & PAOI derivative; $\Delta$ rhIR $\Delta$ lasR, pBADlas $R$ & This study \\
\hline MSCIIO & PAOI derivative; $\Delta r h I R \Delta l a s R, \mathrm{pBAD}$ rhIR & This study \\
\hline $\mathrm{MSCl} 20$ & PAOI derivative; $r p o S:: \mathrm{Gm}^{\mathrm{R}}$, pBADrpoS & This study \\
\hline \multicolumn{3}{|l|}{ Plasmids } \\
\hline pFLP2 & Source of Flp recombinase; $A p^{R}$ & [37] \\
\hline PJNI05 & araC-pBAD cloned in pBBR IMCS-5; GmR & {$[41]$} \\
\hline pJNI05.lasR & lasR in pJNI05 & {$[48]$} \\
\hline PJNI05.rhIR & $r h / R$ in $\mathrm{PJN} 105$ & This study \\
\hline pJNI05.rpos & rpoS in pJNI05 & This study \\
\hline \multirow[t]{2}{*}{ pSWI96 } & mini-CTXI based plasmid containing & {$[40]$} \\
\hline & & araC-pBAD from $\mathrm{pBAD} 30 ; \mathrm{Tc}^{\mathrm{R}}$ \\
\hline pSWI96.lasR & lasR in pSWI96 & This study \\
\hline pSWI96.rhIR & $r h / R$ in $\mathrm{pSW} 196$ & This study \\
\hline pSWI96.rpoS & rpos in pSWI96 & This study \\
\hline
\end{tabular}

construct a double mutant background, a chromosomal las $R$ mutation was introduced into these strains by transformation with chromosomal DNA isolated from a PAO lasR

mutant.

The lasR ORF was amplified from $P$. aeruginosa PAO1 genomic DNA by polymerase chain reaction (PCR) using primers

$\mathrm{N}_{6}$ GAATTCTGATTAACTTTATAAGGAGGAAAACATATG GCCTTGGTTGACGGTTTTC-3' and 5'$\mathrm{N}_{6}$ GCGGCCGCGGCAAGATCAGAGAGTAATAA GAC-3'. The underlined sequences indicate EcoRI and NotI restriction sites, respectively. The sequences in italics indicate a T7 gene enhancer element and an optimized ribosomal binding site (RBS) [39]. These sequences were included to enhance translation of LasR because expression levels from the native RBS (as assessed by Western blotting) were low even when induced fully. The rhlR and rpoS alleles were subcloned from pJN105.rhlR and pJN105.rpoS. These plasmids were constructed previously (see below). The lasR PCR product was cut with EcoRI and NotI, and pJN105.rhlR and pJN105.rpoS were cut with EcoRI-NotI and KpnI-SpeI, respectively. The digested fragments containing lasR, $r h l R$, or $r p o S$ were ligated with appropriately cut pSW196 [40], which contains an arabinose-inducible
araBAD promoter inserted into plasmid mini-CTX1 [38]. The resulting plasmids pSW196.lasR and pSW196.rhlR were each mobilized into PAO rhlR, and pSW196.rpoS was mobilized into PAO rpoS via E. coli S17-1. Mini-CTX1 encodes a site-specific integrase which mediates insertion at the chromosomal attB site, and it also contains Flp recombinase target sites flanking the multiple cloning site thus allowing in vivo excision of unwanted plasmid backbone DNA. To accomplish excision, pFLP2, encoding Flp recombinase, was introduced into the $P$. aeruginos $a$ strains containing the respective lasR, $r h l R$, or $r p o S$ expression constructs. This plasmid was then cured via the pFLP2encoded $s a c B$ counterselectable marker. To determine whether strains were constructed properly, the size of fragments encompassing the $a t t B$ region was determined by PCR with the primers 5'-CGTACAACGTGCCGGATATCG3 ' and 5'GCTTCGGGATAAGCCAATCCTG-3' and subsequent agarose gel electrophoresis. In contrast to lasR and rhlR constructs, the rpoS expression construct did not insert at the $a t t B$ site, even after repeated attempts. It did insert, however, at another, unknown site. We were unable to determine the exact insertion site by arbitrary PCR. We verified that the transcript profile of the rpos mutant carrying the rpos construct under non-inducing conditions was very similar to that of the original rpos mutant, 
indicating that the insertion did not result in significant unintended gene expression changes. Transcription of neighboring chromosomal DNA from the araBAD promoter is minimized by strong $\mathrm{T} 4$ transcriptional terminators [38]. To generate PAO lasR rhlR double mutant backgrounds, a las $:: \mathrm{Tc}^{\mathrm{R}}$ mutation was introduced by transformation of chemically competent cells with $80 \mu \mathrm{g}$ of genomic DNA isolated from PAO lasR::TcR as described [15].

The vectors pJN105.rhlR and pJN105.rpos were constructed as follows: The rhlR and rpoS ORFs including native ribosomal binding sites were amplified from PAO1 genomic DNA by PCR. The primers for rhlR were 5'$\mathrm{N}_{6}$ GAATTCATCGATCAGGGCTTACTGCAATG-3' and 5'$\mathrm{N}_{6}$ TCTAGAGCGCTTCAGATGAGACCCAGC-3' with the underlined sequences indicating EcoRI and $\mathrm{XbaI}$ restriction sites, respectively. The primers for $r p o S$ were $5^{\prime}$ $\mathrm{N}_{6}$ GCTAGCAAGGGATAACGACATGGCAC-3' and 5'$\mathrm{N}_{6}$ GAATTCTCACTGGAACAGCGC GTCACT-3' with the underlined sequences indicating NheI and EcoRI restriction sites, respectively. The resulting PCR fragments were cut with the indicated restriction enzymes and inserted into the multiple cloning site of appropriately digested plasmid pJN105 [41] to give pJN105.rhlR and pJN105.rpoS.

\section{Microarray experiments}

Cultures were harvested at early logarithmic phase $\left(\mathrm{OD}_{600}\right.$ $=0.2)$ and early stationary phase $\left(\mathrm{OD}_{600}=2\right)$. RNA isolation as well as subsequent cDNA synthesis, labeling, and $P$. aeruginosa GeneChip genome array (Affymetrix, Santa Clara, CA) processing were performed as described previously $[8,15]$. Each experiment was done in duplicate. A control experiment confirmed that the addition of arabinose had no significant effect on gene expression. The GeneChip profiles of $P$. aeruginosa in logarithmic phase with and without arabinose showed the same correlation as those of two replicate cultures grown in the absence of arabinose (not shown). Data were processed with the Affymetrix GeneChip Operating Software 1.1. The webbased program Cyber-T [42] was used for statistical analysis as described [15]. The p-value threshold was 0.001, and the corresponding confidence estimate termed posterior probability of differential expression, $\operatorname{PPDE}(<\mathrm{p})$, was $\leq 0.97$. This $p$-value was chosen based on graphical analysis of the distribution of p-values and their association with the non-uniform distribution (indicating differential expression) and the uniform distribution (indicating no differential expression) [42]. To determine which quorum-controlled genes qualify as late genes, we demanded that transcript levels in stationary phase were significantly higher in wildtype $P$. aeruginosa compared to a las $R, r h l R$ mutant, and that this difference was larger in stationary phase than in logarithmic phase. Global gene expression patterns ("heat-maps") were constructed with GeneSpring 7.2 (Agilent Technologies, Palo Alto, CA). Transcript profiles of individual genes were normalized to the median expression level. Microarray data were deposited in the EMBL ArrayExpress repository (Accession number EMEXP-1183).

\section{Real-time PCR}

$P$. aeruginosa culture conditions, as well as RNA isolation and cDNA synthesis procedures were identical to those used for microarray analysis. Real-time PCR was performed with an ABI Prism 7900 Sequence Detection System (Applied Biosystems, Foster City, CA). Cycling parameters were $10 \mathrm{~min}$ at $95^{\circ} \mathrm{C}$ followed by 40 cycles of $15 \mathrm{~s}$ at $95^{\circ} \mathrm{C}$ and $1 \mathrm{~min}$ at $60^{\circ} \mathrm{C}$. Dissociation profiles of the amplified products were run to evaluate non-specific amplification. Each PCR reaction contained $1 \times$ SYBR Green Master Mix (Applied Biosystems), 1 ng cDNA template, and $7.5 \mathrm{pmol}$ of each primer in a $25-\mu$ l volume. Gene-specific primers were designed and data were analyzed using Primer Express and SDS 2.1 software, respectively (Applied Biosystems). Relative transcript levels were determined by using the standard curve method and by using the $n a d B$ transcript as a calibrator. Standard curves were constructed with $10^{-4}$ to $10 \mathrm{pg}$ of RNA-free genomic DNA purified from $P$. aeruginosa PAO1 (Genomic-tip kit, Qiagen). Experiments were performed in duplicate.

\section{Immunoblotting}

For protein analysis, samples were withdrawn from the same $P$. aeruginosa cultures that were used for transcript profiling. Cells were harvested by centrifugation. Pellets were suspended in lysis buffer containing $25 \mathrm{mM}$ Tris$\mathrm{HCl}, \mathrm{pH} 7.8,150 \mathrm{mM} \mathrm{NaCl}, 1 \mathrm{mM}$ EDTA, $1 \mathrm{mM}$ dithiothreitol, $0.5 \%$ Tween-20, 10\% glycerol, $2 \mu \mathrm{M}$ 3OC12-HSL and $10 \mu \mathrm{M}$ C4-HSL. The suspensions were sonicated, and the resulting lysates subjected to ultracentrifugation at $100,000 \times \mathrm{g}$ for $15 \mathrm{~min}$. Protein concentrations were determined by using the Bradford assay. Approximately 2 $\mu \mathrm{g}$ of each supernatant fraction was separated by $12.5 \%$ SDS-PAGE. The separated proteins were blotted onto a nylon membrane. The membrane was treated with polyclonal antibodies against LasR, RhlR, or RpoS. Proteins were detected by using a secondary anti-rabbit horseradish peroxidase-conjugated IgG and chemiluminescent substrate (Pierce, Rockford, IL). Antibodies against P. aeruginosa LasR and RhlR were generated in rabbits immunized with $\mathrm{His}_{10}$-LasR or $\mathrm{His}_{10}$-RhlR affinity-purified under denaturing conditions. Polyclonal rabbit antibody against $P$. aeruginosa RpoS was obtained from V. Venturi [43].

\section{Consensus sequence analysis}

Ten predicted promoter regions (-400 to - 1 relative to the translational start) associated with the genes most highly 
induced by either LasR alone or by RhlR alone (Additional file 1) were each subjected to the pattern search algorithm CONSENSUS [28]. Overlap of sequences with upstream open reading frames was disallowed. A matrix length of 16 bp was chosen for the final alignment, because it yielded the most consistent results. The highest scoring matrix from the final cycle was used to depict a sequence alignment in WEBLOGO [44]. The same matrix was also used in PATSER [28] to identify sequences in upstream regulatory regions of other quorum-controlled genes. The default weight score of 7 was used as the lower threshold.

\section{Authors' contributions}

MS designed the study, carried out experiments and drafted the manuscript. EPG conceived of the study, participated in its design and coordination and helped to draft the manuscript. All authors read and approved the final manuscript.

\section{Additional material}

\section{Additional file 1}

Table S1. Quorum-controlled genes that can be advanced by signal addition or LasR, RhlR or RpoS overexpression. This table lists P. aeruginosa quorum-controlled genes that are expressed early in growth when acylHSL signals are added or when LasR, RhlR, or RpoS are expressed ectopically.

Click here for file

[http://www.biomedcentral.com/content/supplementary/1471-

2164-8-287-S1.xls]

\section{Additional file 2}

Table S2. Late quorum-controlled genes that cannot be advanced by signal addition or LasR, RhlR, or RpoS overexpression. This table lists P. aeruginosa quorum-controlled genes that are not expressed early in growth even when acyl-HSL signals are added or when LasR, RhlR, or RpoS are expressed ectopically.

Click here for file

[http://www.biomedcentral.com/content/supplementary/14712164-8-287-S2.xls]

\section{Acknowledgements}

We thank Esther Volper and the University of lowa DNA Facility for microarray processing. We thank Milica Sevo and Vittorio Venturi for providing anti-RpoS antibody. We also thank Dan Wozniak and Herbert Schweitzer for providing the integration-proficient plasmids. This work was supported by USPHS grant GM-59026.

\section{References}

I. Parsek MR, Greenberg EP: Acyl-homoserine lactone quorum sensing in gram-negative bacteria: a signaling mechanism involved in associations with higher organisms. Proc Natl Acad Sci USA 2000, 97:8789-8793.

2. Fuqua $C$, Greenberg EP: Listening in on bacteria: acyl-homoserine lactone signalling. Nat Rev Mol Cell Biol 2002, 3(9):685-695

3. Smith RS, Iglewski $B H: P$. aeruginosa quorum-sensing systems and virulence. Curr Opin Microbiol 2003, 6(I):56-60.
4. Whitehead NA, Barnard AM, Slater H, Simpson NJ, Salmond GP: Quorum-sensing in Gram-negative bacteria. FEMS Microbiol Rev 200I, 25(4):365-404.

5. Juhas M, Eberl L, Tummler B: Quorum sensing: the power of cooperation in the world of Pseudomonas. Environ Microbiol 2005, 7(4):459-47I.

6. Hentzer M, Wu H, Andersen JB, Riedel K, Rasmussen TB, Bagge N, Kumar N, Schembri MA, Song Z, Kristoffersen P, Manefield M, Costerton JW, Molin S, Eberl L, Steinberg P, Kjelleberg S, Hoiby N, Givskov M: Attenuation of Pseudomonas aeruginosa virulence by quorum sensing inhibitors. $E M B O \mathrm{~J}$ 2003, 22(15):3803-38I5.

7. Wagner VE, Bushnell D, Passador L, Brooks Al, Iglewski BH: Microarray analysis of Pseudomonas aeruginosa quorum-sensing regulons: effects of growth phase and environment. J Bacteriol 2003, I 85(7):2080-2095.

8. Schuster M, Lohstroh CP, Ogi T, Greenberg EP: Identification, timing and signal specificity of Pseudomonas aeruginosa quorumcontrolled genes: A transcriptome analysis. J Bacteriol 2003, 185:2066-2079.

9. Winzer K, Falconer C, Garber NC, Diggle SP, Camara M, Williams P. The Pseudomonas aeruginosa lectins PA-IL and PA-IIL are controlled by quorum sensing and by RpoS. J Bacteriol 2000 , I 82(22):640 I-64II.

10. Diggle SP, Winzer K, Lazdunski A, Williams P, Camara M: Advancing the quorum in Pseudomonas aeruginosa: MvaT and the regulation of $\mathbf{N}$-acylhomoserine lactone production and virulence gene expression. J Bacteriol 2002, 184(10):2576-2586.

II. Whiteley M, Lee KM, Greenberg EP: Identification of genes controlled by quorum sensing in Pseudomonas aeruginosa. Proc Natl Acad Sci USA 1999, 96:13904-13909.

12. Heurlier K, Williams F, Heeb S, Dormond C, Pessi G, Singer D, Camara M, Williams P, Haas D: Positive control of swarming, rhamnolipid synthesis, and lipase production by the posttranscriptional RsmA/RsmZ system in Pseudomonas aeruginosa PAOI. J Bacteriol 2004, I 86( I 0):2936-2945.

13. Pessi G, Williams F, Hindle Z, Heurlier K, Holden MT, Camara M, Haas D, Williams $P$ : The global posttranscriptional regulator RsmA modulates production of virulence determinants and $\mathbf{N}$-acylhomoserine lactones in Pseudomonas aeruginosa. J Bacteriol 200I, I 83(22):6676-6683.

14. Reimmann C, Beyeler M, Latifi A, Winteler H, Foglino M, Lazdunski A, Haas D: The global activator GacA of Pseudomonas aeruginosa PAO positively controls the production of the autoinducer $\mathrm{N}$-butyryl-homoserine lactone and the formation of the virulence factors pyocyanin, cyanide, and lipase. Mol Microbiol 1997, 24(2):309-319.

15. Schuster M, Hawkins AC, Harwood CS, Greenberg EP: The Pseudomonas aeruginosa $\mathrm{RpoS}$ regulon and its relationship to quorum sensing. Mol Microbiol 2004, 5 I (4):973-985.

16. Pesci EC, Milbank JB, Pearson JP, McKnight S, Kende AS, Greenberg $\mathrm{EP}$, Iglewski BH: Quinolone signaling in the cell-to-cell communication system of Pseudomonas aeruginosa. Proc Natl Acad Sci USA 1999, 96(20): I I229-I I 234.

17. Deziel E, Gopalan S, Tampakaki AP, Lepine F, Padfield KE, Saucier M, Xiao G, Rahme LG: The contribution of MvfR to Pseudomonas aeruginosa pathogenesis and quorum sensing circuitry regulation: multiple quorum sensing-regulated genes are modulated without affecting lasRI, rhIRI or the production of $\mathbf{N}$ acyl-L-homoserine lactones. Mol Microbiol 2005, 55(4):998-1014.

18. Schuster M, Greenberg EP: A network of networks: Quorum sensing gene regulation in Pseudomonas aeruginosa. Int J Med Microbiol 2006, 296:73-8I.

19. Yarwood JM, Volper EM, Greenberg EP: Delays in Pseudomonas aeruginosa quorum-controlled gene expression are conditional. Proc Natl Acad Sci USA 2005, I 02(25):9008-90I 3.

20. Pesci EC, Pearson JP, Seed PC, Iglewski BH: Regulation of las and rhl quorum sensing in Pseudomonas aeruginosa. J Bacteriol I997, I 79(10):3।27-3132.

21. Albus AM, Pesci EC, Runyen-Janecky LJ, West SE, Iglewski BH: Vfr controls quorum sensing in Pseudomonas aeruginosa. J Bacteriol 1997, I 79( I 2):3928-3935.

22. Whiteley M, Parsek MR, Greenberg EP: Regulation of quorum sensing by RpoS in Pseudomonas aeruginosa. J Bacteriol 2000, I 82( (15):4356-4360. 
23. Latifi A, Foglino M, Tanaka K, Williams P, Lazdunski A: A hierarchical quorum-sensing cascade in Pseudomonas aeruginosa links the transcriptional activators LasR and RhIR (VsmR) to expression of the stationary-phase sigma factor RpoS. Mol Microbiol 1996, 2 I(6): I |37-I|46.

24. Medina G, Juarez K, Diaz R, Soberon-Chavez G: Transcriptional regulation of Pseudomonas aeruginosa rhIR, encoding a quorum-sensing regulatory protein. Microbiology 2003, I49(Pt I I):3073-308I.

25. Pearson JP, Pesci EC, Iglewski BH: Roles of Pseudomonas aeruginosa las and rhl quorum-sensing systems in control of elastase and rhamnolipid biosynthesis genes. J Bacteriol 1997 I 79( I 8):5756-5767.

26. Schuster M, Greenberg EP: A network of networks: quorumsensing gene regulation in Pseudomonas aeruginosa. Int J Med Microbiol 2006, 296(2-3):73-8I.

27. Li LL, Malone JE, Iglewski BH: Regulation of the Pseudomonas aeruginosa quorum-sensing regulator VqsR. J Bacteriol 2007.

28. van Helden J: Regulatory sequence analysis tools. Nucleic Acids Res 2003, 3 I ( I 3):3593-3596.

29. Schuster M, Urbanowski ML, Greenberg EP: Promoter specificity in Pseudomonas aeruginosa quorum sensing revealed by DNA binding of purified LasR. Proc Natl Acad Sci USA 2004 I0I:I5833-I5839.

30. Whiteley M, Greenberg EP: Promoter specificity elements in Pseudomonas aeruginosa quorum-sensing-controlled genes. J Bacteriol 2001, I 83( I 9):5529-5534.

31. Medina G, Juarez K, Soberon-Chavez G: The Pseudomonas aeruginosa rhIAB operon is not expressed during the logarithmic phase of growth even in the presence of its activator RhIR and the autoinducer $\mathbf{N}$-butyryl-homoserine lactone. J Bacteriol 2003, I 85(I):377-380.

32. Shen-Orr SS, Milo R, Mangan S, Alon U: Network motifs in the transcriptional regulation network of Escherichia coli. Nat Genet 2002, 3 I (I):64-68.

33. Kay E, Humair B, Denervaud V, Riedel K, Spahr S, Eberl L, Valverde C, Haas D: Two GacA-dependent small RNAs modulate the quorum-sensing response in Pseudomonas aeruginosa. J Bacteriol 2006, I 88(16):6026-6033.

34. Sonnleitner E, Schuster M, Sorger-Domenigg T, Greenberg EP, Blasi $\mathrm{U}$ : Hfq-dependent alterations of the transcriptome profile and effects on quorum sensing in Pseudomonas aeruginosa. Mol Microbiol 2006, 59(5): I 542-I558.

35. Vasil ML: DNA microarrays in analysis of quorum sensing: strengths and limitations. I Bacteriol 2003, I 85(7):206 |-2065.

36. Buck MJ, Lieb JD: ChIP-chip: considerations for the design, analysis, and application of genome-wide chromatin immunoprecipitation experiments. Genomics 2004, 83(3):349-360.

37. Hoang TT, Karkhoff-Schweizer RR, Kutchma AJ, Schweizer HP: A broad-host-range FIp-FRT recombination system for sitespecific excision of chromosomally-located DNA sequences: application for isolation of unmarked Pseudomonas aeruginosa mutants. Gene 1998, 28:77-86.

38. Hoang TT, Kutchma AJ, Becher A, Schweizer HP: Integration-proficient plasmids for Pseudomonas aeruginosa: site-specific integration and use for engineering of reporter and expression strains. Plasmid 2000, 43:59-7I.

39. Miller WG, Lindow SE: An improved GFP cloning cassette designed for prokaryotic transcriptional fusions. Gene 1997, I91(2): | $49-153$

40. Baynham PJ, Ramsey DM, Gvozdyev BV, Cordonnier EM, Wozniak DJ: The Pseudomonas aeruginosa ribbon-helix-helix DNAbinding protein AlgZ (AmrZ) controls twitching motility and biogenesis of type IV pili. J Bacteriol 2006, I 88( I): |32-|40.

4I. Newman JR, Fuqua C: Broad-host-range expression vectors that carry the L-arabinose-inducible Escherichia coli araBAD promoter and the araC regulator. Gene 1999, 227(2):197-203.

42. Hatfield GW, Hung SP, Baldi P: Differential analysis of DNA microarray gene expression data. Mol Microbiol 2003, 47(4):87I-877.

43. Kojic M, Venturi V: Regulation of rpoS gene expression in Pseudomonas: involvement of a TetR family regulator. I Bacterio 200I, I 83(I 2):37I 2-3720.

44. Crooks GE, Hon G, Chandonia JM, Brenner SE: WebLogo: a sequence logo generator. Genome Res 2004, I 4(6): I | 88- | | 90.
45. Simon $\mathrm{R}$, Priefer $\mathrm{V}$, Puhler A: A broad host range mobilisation system for in vivo genetic engineering: transposon mutagenesis in gram negative bacteria. Biotechnology 1983, I:784-791.

46. Holloway JB, Krishnapillai V, Morgan AF: Chromosomal genetics of Pseudomonas. Microbiol Rev 1979, 43:73-102.

47. Rahim R, Ochsner UA, Olvera C, Graninger M, Messner P, Lam JS, Soberon-Chavez G: Cloning and functional characterization of the Pseudomonas aeruginosa rhIC gene that encodes rhamnosyltransferase 2, an enzyme responsible for di-rhamnolipid biosynthesis. Mol Microbiol 200I, 40(3):708-7I8.

48. Lee JH, Lequette $Y$, Greenberg EP: Activity of purified QscR, a Pseudomonas aeruginosa orphan quorum-sensing transcription factor. Mol Microbiol 2006, 59(2):602-609.
Publish with Biomed Central and every scientist can read your work free of charge

"BioMed Central will be the most significant development for disseminating the results of biomedical research in our lifetime. "

Sir Paul Nurse, Cancer Research UK

Your research papers will be:

- available free of charge to the entire biomedical community

- peer reviewed and published immediately upon acceptance

- cited in PubMed and archived on PubMed Central

- yours - you keep the copyright
BioMedcentral 